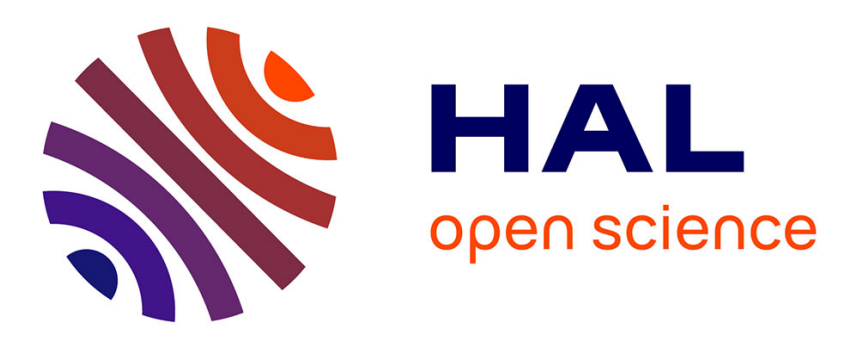

\title{
Ketone physics - structure, conformations, and dynamics of methyl isobutyl ketone explored by microwave spectroscopy and quantum chemical calculations
}

\author{
Yueyue Zhao, Wolfgang Stahl, Ha Vinh Lam Nguyen
}

\section{To cite this version:}

Yueyue Zhao, Wolfgang Stahl, Ha Vinh Lam Nguyen. Ketone physics - structure, conformations, and dynamics of methyl isobutyl ketone explored by microwave spectroscopy and quantum chemical calculations. Chemical Physics Letters, 2012, 545, pp.9-13. 10.1016/j.cplett.2012.07.009 . hal-03183164

\author{
HAL Id: hal-03183164 \\ https://hal.science/hal-03183164
}

Submitted on 27 Mar 2021

HAL is a multi-disciplinary open access archive for the deposit and dissemination of scientific research documents, whether they are published or not. The documents may come from teaching and research institutions in France or abroad, or from public or private research centers.
L'archive ouverte pluridisciplinaire $\mathbf{H A L}$, est destinée au dépôt et à la diffusion de documents scientifiques de niveau recherche, publiés ou non, émanant des établissements d'enseignement et de recherche français ou étrangers, des laboratoires publics ou privés. 


\title{
Ketone physics - structure, conformations, and dynamics of methyl isobutyl ketone explored by microwave spectroscopy and quantum chemical calculations
}

\author{
Yueyue Zhao, Wolfgang Stahl, and Ha Vinh Lam Nguyen ${ }^{\mathrm{a}}$ \\ ${ }^{\text {a }}$ Institute of Physical Chemistry, RWTH Aachen University, Landoltweg 2, D-52074 Aachen, \\ Germany \\ Corresponding author: Ha Vinh Lam Nguyen \\ Institute of Physical Chemistry \\ RWTH Aachen University \\ Landoltweg 2 \\ D-52074 Aachen \\ Germany \\ Phone: +492418094759 \\ Fax: +492418092365 \\ Email: nguyen@pc.rwth-aachen.de
}

\begin{abstract}
The microwave spectrum of methyl isobutyl ketone was recorded using a molecular beam Fourier transform microwave spectrometer and analyzed to determine the rotational constants $\mathrm{A}=4.7517(17) \mathrm{GHz}, \mathrm{B}=1.496115(79) \mathrm{GHz}, \mathrm{C}=1.324364(39) \mathrm{GHz}$, and centrifugal distortion constants. A-E splittings from $250 \mathrm{MHz}$ up to $1 \mathrm{GHz}$ were observed due to internal rotation of the acetyl methyl group with a potential barrier of $250.3(19) \mathrm{cm}^{-1} .59 \mathrm{~A}$ and $57 \mathrm{E}$ species lines were fitted to experimental accuracy. The experimental rotational constants were compared with results from quantum chemical calculations to validate the molecular structure.
\end{abstract}

Keywords: methyl isobutyl ketone, microwave spectroscopy, internal rotation, quantum chemical calculation

\section{Introduction}

Methyl isobutyl ketone is a widely used polar solvent and extracting agent for gold, silver, and other rare elements from cyanide solutions in chemistry and industry. It is not only one of the most important ketones, but also interesting due to its structure and dynamics. The rotational spectrum of the simplest ketone, acetone, has been treated by many previous investigations, first in 1959 by Swalen and Costain [1], afterwards by Peter and Dreizler [2]. Two larger aliphatic ketones, methyl vinyl ketone and methyl ethyl ketone, were also studied. The methyl groups of acetone, methyl vinyl ketone, and methyl ethyl ketone show internal rotation with quite different barriers of $264.7(8) \mathrm{cm}^{-1}$ [2], 424(7) $\mathrm{cm}^{-1}$ [3], and 183(5) $\mathrm{cm}^{-1}$ [4], respectively. It is interesting to determine the barrier to internal rotation of the methyl group of methyl isobutyl ketone and to compare it with the values found for other aliphatic ketones.

From the investigation on isopropyl acetate [5] we know that its most stable conformer has no symmetry plane. One isopropyl methyl group is located in the C-COO plane and the other is 
tilted out. We were interested in comparing the location of the isopropyl group of methyl isobutyl ketone with isopropyl acetate since the structures of these molecules are very similar, except that the isopropoxy oxygen is exchanged by a $\mathrm{CH}_{2}$ group.

Methyl isobutyl ketone is a molecule with 19 atoms $\left(\mathrm{C}_{6} \mathrm{H}_{12} \mathrm{O}\right)$, which is too large for classical structure determination by isotopic substitution, but even larger molecular systems can nowadays be calculated with accurate methods and large basis sets. Those molecular structures can be validated by comparing calculated spectroscopic parameters with experimentally determined ones. The quantum chemical results are reasonable in many cases we studied before like ethyl acetate [6], isopropenyl acetate [7], ethyl valerate [8], and methyl propionate [9]. However, in the case of diethyl ketone [10], calculations using two different methods MP2 and DFT gave quite different molecular structures for the same basis set. Therefore, it is interesting to find out, whether in the case of methyl isobutyl ketone the quantum chemical calculations are reliable.

\section{Experimental setup}

The investigation on methyl isobutyl ketone was carried out by molecular beam Fourier transform microwave spectroscopy. Methyl isobutyl ketone was obtained from Merck Schuchardt OHG, Hohenbrunn, Germany. A mixture of $1 \%$ by volume of methyl isobutyl ketone in helium was prepared and expanded into the Fabry-Perot resonator of the spectrometer. The rotational temperature is approximately $1.5 \mathrm{~K}$.

The spectrometer used to record the spectrum is a modified version of that described in ref. [11] and therefore, the construction will not be repeated here. Two different modes can be used, the high resolution mode and the scan mode. In the high resolution mode, all lines split into doublets due to the Doppler effect. The line widths depend on the respective transitions and vary from 9 to $22 \mathrm{kHz}$. This might be attributed to unresolved splittings due to the spinspin and spin-rotation coupling from the 12 protons in the molecule. A typical spectrum in the high resolution mode is shown in Figure 1. In the scan mode, overlapping spectra are automatically recorded and only the line positions are indicated.

\section{Quantum chemical calculations}

Before beginning the experimental investigations, quantum chemical calculations were carried out to get some idea of the structure of methyl isobutyl ketone. By varying the two dihedral angles $\varphi_{1}=\angle\left(\mathrm{C}_{1}, \mathrm{C}_{7}, \mathrm{C}_{10}, \mathrm{C}_{12}\right)$ and $\varphi_{2}=\angle\left(\mathrm{O}_{2}, \mathrm{C}_{1}, \mathrm{C}_{7}, \mathrm{C}_{10}\right), 16$ starting geometries were created and optimized with the program Gaussian09 [12] using two different methods MP2 and B3LYP with the 6-311++G(d,p) basis set; for the atom numbering see Figure 2. Four stable conformers were found and indicated in Figure 3 with their energies relative to the most stable conformer I. Conformer II is close to $\mathrm{C}_{\mathrm{s}}$ symmetry with the $\mathrm{C}-(\mathrm{CO})-\mathrm{C}$ plane serving as the "mirror plane". However, this "mirror plane" does not coincide with the $a b$ or the $a c$ plane, but it is located somewhere in between, and pretty strong dipole moment components are predicted for all principal axes. The reason is that the $\mathrm{B}$ and $\mathrm{C}$ rotational constants are very similar, and conformer II is by accident very close to a prolate symmetric top. In a real prolate top the $b$ and $c$ axes are mutually perpendicular to each other, but they may be arbitrarily rotated within a plane perpendicular to the $a$ axis. Therefore in conformer II, the small deviation from perfect $\mathrm{C}_{\mathrm{s}}$ symmetry determines, where the $b$ and the $c$ axis are located. All other conformers have structures with no symmetry $\left(C_{1}\right)$ where either the isopropyl methyl group or the complete isopropyl group is tilted out of the $\mathrm{C}-(\mathrm{CO})-\mathrm{C}$ plane. They always 
appear as enantiomeric pairs. The rotational constants, energies, dipole moments, as well as the Cartesian coordinates are given as supplementary material in Table S-1.

The most stable conformer obtained for methyl isobutyl ketone by quantum chemical calculations has a very similar structure as the observed conformer of isopropyl acetate [5]. In addition to structure optimizations for all conformers, two minimum potential energy paths (MPEP) were calculated. In one case, the isopropyl group was rotated by varying the angle $\varphi_{1}$ at a step width of $10^{\circ}$, while all other parameters were optimized. The potential curve is shown in Figure 4, the Fourier coefficients of the parameterized curve are given in Table S-2. In the region from 0 to $240^{\circ}$ an almost symmetric double minimum potential is found. The minima at $\varphi_{1}=170^{\circ}$ and $70^{\circ}$ belong to conformer I and its enantiomer I*, respectively. A third minimum around $\varphi_{1}=300^{\circ}$ represents conformer II.

In a second case, the isobutyl group was rotated by variation of angle $\varphi_{2}$ in steps of $10^{\circ}$. The MPEP is presented in Figure 5; the Fourier coefficients are also given in Table S-2. The curve is completely asymmetric. This time conformer I is found at $\varphi_{2}=340^{\circ}$, corresponding to a geometry where the isopropyl group is tilted out of the C-(CO)-C plane by approximately $20^{\circ}$. In the optimized conformer I shown in Figure 2, the tilt angles calculated with the MP2 and B3LYP methods are $20.2^{\circ}$ and $15.6^{\circ}$, respectively.

It should be noted that in both, Figure 3 and Figure 5, the energies of conformer III calculated with the MP2 and B3LYP method are quite different. This is in agreement with our experience from similar studies on diethyl ketone [10], methyl propionate [9], ethyl isovalerate [13], and ethyl valerate [8]. In those cases the energies of some conformers were also strongly dependent on the method of calculation. Conformer IV is missing in both MPEPs. The reason is that if angle $\varphi_{1}$ is fixed to a value corresponding to conformer IV after relaxation of all other parameters, conformer I, which has a similar $\varphi_{1}$ but which is lower in energy, is obtained. A similar situation is encountered when $\varphi_{2}$ is fixed to the value of conformer IV.

In conclusion, conformer I has the lowest energy, and it should give the strongest spectrum under molecular beam conditions. Conformer III has a slightly higher energy. However, the difference is only $1.05 \mathrm{~kJ} / \mathrm{mol}$ (calculated with the MP2 method). Therefore, we expected that this conformer could also be observed in the microwave spectrum.

\section{Spectrum assignment}

At the beginning of our experimental work, two broadband scans in the frequency range from 11.0 to $12.7 \mathrm{GHz}$ and from 15.1 to $17.0 \mathrm{GHz}$ with 134 lines were recorded, as given in Figure 6 . The methyl group in methyl isobutyl ketone shows internal rotation, which causes all lines in the spectrum to split into A-E doublets. We started our assignment with conformer I and used the calculated rotational constants to predict the A species spectrum, which can be considered as a semi-rigid rotor spectrum, where we allow for centrifugal distortion. $59 \mathrm{~A}$ species lines could be assigned and fitted to determine the rotational constants and centrifugal distortion constants. The assigned A species lines in the frequency range from 15.1 to 17.0 $\mathrm{GHz}$ are indicated as black lines in the lower trace of Figure 6.

After the semi-rigid rotor rotational constants were determined, we assumed the barrier to internal rotation of the acetyl methyl group to be approximately the same as in methyl ethyl ketone, where it is $183 \mathrm{~cm}^{-1}$, and predicted the complete rotational spectrum using the 
program XIAM [14]. After varying the internal rotation barrier by trial and error, the predicted E species transitions finally matched exactly the measured spectrum. The lower trace of Figure 6 shows a $c$-type Q branch, which was helpful for the assignment of the spectrum. In total, $59 \mathrm{~A}$ species and $57 \mathrm{E}$ species transitions found in the broadband scans were assigned to conformer I. The line positions without line intensities are also indicated in the lower trace of Figure 6. These lines were fitted with XIAM to a standard deviation of 16.3 $\mathrm{kHz}$. A barrier to internal rotation of $250.5 \mathrm{~cm}^{-1}$ was found, which is surprisingly similar to the value found for acetone rather than that of methyl ethyl ketone.

The data set was fitted again using the program Erham, another code treating internal rotation written by Groner [15]. The fitted parameters are given in Table 1 and 2. The frequency list and the observed-minus-calculated values of all transitions fitted by XIAM and Erham are available in Table S-3 in the supplementary material. Whereas the XIAM code uses the Internal Axis Method (IAM), Erham sets up and solves an Effective Rotational HAMiltonian. Details of the Hamiltonian are extensively discussed in [14] and [15]. In the case of methyl isobutyl ketone, the effective Hamiltonian gives a better standard deviation since more higher order teams can be included in the fit.

\section{Results and discussion}

116 transitions of conformer I ( $\mathrm{C}_{1}$ symmetry) in the microwave spectrum of methyl isobutyl ketone could be assigned and fitted with two different program codes, XIAM and Erham. Using the program XIAM, rotational constants, centrifugal distortion constants, and three higher order terms $\mathrm{D}_{\mathrm{pi} 2 \mathrm{~J}}, \mathrm{D}_{\mathrm{pi} 2 \mathrm{~K}}$, and $\mathrm{D}_{\mathrm{pi} 2-}$, as well as the barrier to internal rotation and the angles between the internal rotor axis and the principal axes could be fitted with very high accuracy. The definitions of $D_{\text {pi2J, }}, D_{\text {pi2K, }}$, and $D_{\text {pi2- }}$ are given in [18], equation 6 , with $D_{\text {pi2J }}=$ $\Delta_{\mathrm{Jm}}, \mathrm{D}_{\mathrm{pi} 2 \mathrm{~K}}=\Delta_{\mathrm{Km}}$, and $\mathrm{D}_{\mathrm{pi} 2^{-}}=\delta_{\mathrm{m}}$. They can be considered as centrifugal distortion terms involving the angular momentum of the methyl rotor. The differences between the calculated and experimental $\mathrm{A}, \mathrm{B}$, and $\mathrm{C}$ rotational constants are within $1 \%$. Obviously, the calculated rotational constants at the MP2/6-311++G(d,p) level of theory were reasonable starting values and turned out to be quite helpful for the spectral assignment.

The barrier to internal rotation of $250.3 \mathrm{~cm}^{-1}$ is almost the same as the value of $265 \mathrm{~cm}^{-1}$ found for acetone. It should be noted that the substituent on the other side of the carbonyl groups has a large effect on the barrier to internal rotation of the acetyl methyl group. Whereas the barriers are very low and almost always around $100 \mathrm{~cm}^{-1}$ in the cases of most acetates (acetic acid esters), the acetyl methyl group in methyl ethyl ketone, $s p$-methyl vinyl ketone, and $a p$ methyl vinyl ketone are $183 \mathrm{~cm}^{-1}$ [4], $377 \mathrm{~cm}^{-1}$ [19], and $434 \mathrm{~cm}^{-1}$ [19], respectively. The considerably higher barrier in the two conformers of methyl vinyl ketone can be explained with the delocalization of the $\pi$-electrons which conjuncate from the vinyl double bond to the carbonyl group. A similar case was discussed in ref. [7], since the barriers found for isopropenyl acetate $\left(135 \mathrm{~cm}^{-1}\right)$ and vinyl acetate $\left(158 \mathrm{~cm}^{-1}\right)$ are higher than the usual value of $100 \mathrm{~cm}^{-1}$ found for all other investigated acetate esters like methyl acetate [20], ethyl acetate [6], isopropyl acetate [5], and allyl acetate [21]. The barrier found for acetone is higher than in methyl ethyl ketone. This might be due to the strong interaction between two methyl groups in acetone. Since no considerable interaction is expected for methyl isobutyl ketone, it was surprising that the barrier is almost the same as in acetone, whereas a similar barrier as in methyl ethyl ketone was expected. 
The standard deviation of the fit is $16.3 \mathrm{kHz}$ using the program XIAM. This is higher than our experimental accuracy. As discussed in ref. [6], [7], and [21], the results obtained with XIAM are not completely satisfactory if low barrier internal rotation is present. Unfortunately, the internal rotation barrier of methyl isobutyl keton is rather low if compared to the barriers found for the methoxy methyl groups of methyl acetate $\left(422 \mathrm{~cm}^{-1}\right)$ [20] and methyl propionate $\left(429 \mathrm{~cm}^{-1}\right)$ [9] and the ethyl methyl groups of diethyl ketone $\left(772 \mathrm{~cm}^{-1}\right)$ [10], ethyl methyl ketone $\left(795 \mathrm{~cm}^{-1}\right)$ [4], methyl propionate $\left(820 \mathrm{~cm}^{-1}\right)$ [9], and ethyl acetate $\left(1060 \mathrm{~cm}^{-1}\right)$ [6]. Therefore, it could not be expected that the spectrum of methyl isobutyl ketone can be fitted to experimental accuracy. In order to check the assignment, the whole data set was fitted again with the program Erham to an excellent standard deviation of $1.4 \mathrm{kHz}$, which is within the experimental accuracy. We thus conclude that the assignment using the predictions from XIAM is correct. However, as discussed in ref. [7], some parameters fitted with Erham are effective and their physical meanings are less clear than those of XIAM. Furthermore, it is difficult to extract the barrier to internal rotation from the Erham fit.

Finally, it should be noted that 116 lines from 134 lines in the two broadband scans could be assigned to conformer I and only 18 weak lines remain unassigned. Therefore, we conclude that conformer I is the only conformer of methyl isobutyl ketone, which can be observed under molecular beam conditions. Unassigned lines could arise from impurities or ${ }^{13} \mathrm{C}$ isotopologues of the strongest lines of conformer I.

\section{Acknowledgement}

We thank X. Chen for her contribution within her bachelor thesis. Furthermore, we thank the center for Computing and Communication of the RWTH Aachen University for free computer time and the Land Nordrhein-Westfalen for funds. We are also indebted to P. Groner for making his code available for the spectroscopic community.

\section{References}

[1] J. D. Swalen, C. C. Costain, J. Chem. Phys. 31 (1959) 1562.

[2] R. Peter, H. Dreizler, Z. Naturforsch. 204 (1965) 301.

[3] A.C. Fantoni, W. Caminati, R. Meyer, Chem. Phys. Lett. 133 (1987) 27.

[4] N. M. Pozdeev, A. K. Mamleev, L. N. Gunderova, R. V. Galeev, J. Struc. Chem. 29 (1988) 52.

[5] H. Mouhib, D. Jelisavac, W. Stahl, R. Wang, I. Kalf, U. Englert, ChemPhysChem 12

(2011) 761.

[6] D. Jelisavac, D.C. Cortes-Gomez, H.V.L. Nguyen, L.W. Sutikdja, W. Stahl, and I. Kleiner, J. Mol. Specstrosc. 257 (2009) 111.

[7] H.V.L. Nguyen and W. Stahl, J. Mol. Spec. 264 (2010) 120.

[8] H. Mouhib and W. Stahl, ChemPhysChem 13 (2012) 1297.

[9] H.V.L. Nguyen, W. Stahl, I. Kleiner, Mol. Phys. 2012, to be pulished.

[10] H.V.L. Nguyen and W. Stahl, Chem. Phys. Chem. 12 (2011) 1900.

[11] U. Andresen, H. Dreizler, J.-U. Grabow, W. Stahl, Rev. Sci. Instrum. 61 (1990) 3694.

[12] Gaussian 09, Revision A.02, M.J. Frisch, G.W. Trucks, H.B. Schlegel, G.E. Scuseria,

M.A. Robb, J.R. Cheeseman, G. Scalmani, V. Barone, B. Mennucci, G.A. Petersson, H.

Nakatsuji, M. Caricato, X. Li, H.P. Hratchian, A.F. Izmaylov, J. Bloino, G. Zheng, J.L.

Sonnenberg, M. Hada, M. Ehara, K. Toyota, R. Fukuda, J. Hasegawa, M. Ishida, T. Nakajima, 
Y. Honda, O. Kitao, H. Nakai, T. Vreven, J.A. Montgomery, Jr., J.E. Peralta, F. Ogliaro, M. Bearpark, J.J. Heyd, E. Brothers, K.N. Kudin, V.N. Staroverov, R. Kobayashi, J. Normand, K. Raghavachari, A. Rendell, J.C. Burant, S.S. Iyengar, J. Tomasi, M. Cossi, N. Rega, J. M.

Millam, M. Klene, J.E. Knox, J.B. Cross, V. Bakken, C. Adamo, J. Jaramillo, R. Gomperts, R. E. Stratmann, O. Yazyev, A.J. Austin, R. Cammi, C. Pomelli, J.W. Ochterski, R.L. Martin, K. Morokuma, V.G. Zakrzewski, G.A. Voth, P. Salvador, J.J. Dannenberg, S. Dapprich, A.D. Daniels, O. Farkas, J.B. Foresman, J.V. Ortiz, J. Cioslowski, D.J. Fox, Gaussian, Inc., Wallingford CT, 2009.

[13] H. Mouhib, D. Jelisavac, L.W. Sutikdja, E. Isaak, W. Stahl, J. Phys. Chem. A 115 (2011) 118.

[14] H. Hartwig and H. Dreizler, Z. Naturforsch. 51a (1996) 923.

[15] P. Groner, J. Chem. Phys. 107 (1997) 4483.

[16] P. Groner, S. Albert, E. Herbst, F. C. D. Lucia, F. J. Lovas, B. J. Drouin, and J. C. Pearson, ApJS 142 (2002) 145.

[17] P. Groner, Instructions for program ERHAM, available at http://www.ifpan.edu.pl/ kisiel/prospe.htm.

[18] N. Hansen, H. Mäder, T. Bruhn, Mol. Phys. 97 (1999) 587.

[19] D.S. Wilcox, A.J. Shirar, O.L. Williams, B.C. Dian, Chem. Phys. Lett. 508 (2011) 10.

[20] M. Tudorie, I. Kleiner, J. T. Hougen, S. Melandri, L. W. Sutikdja, W. Stahl, J. Mol. Spectrosc. 269 (2011) 211.

[21] H.V.L. Nguyen, H. Mouhib, W. Stahl, and I. Kleiner, Mol. Phys. 108 (2010) 763. 
Table 1:

\begin{tabular}{|c|c|c|c|c|c|}
\hline Parameter & Unit & XIAM & Erham & Calc. $^{\mathrm{a}}$ & Exp. ${ }^{b}-$ Calc \\
\hline A & $\mathrm{GHz}$ & $4.7517(17)$ & $4.74360(29)$ & 4.755 & -0.004 \\
\hline B & $\mathrm{GHz}$ & $1.496115(79)$ & $1.495314(62)$ & 1.506 & -0.010 \\
\hline $\mathrm{C}$ & $\mathrm{GHz}$ & $1.324364(39)$ & $1.327545(68)$ & 1.336 & -0.012 \\
\hline$\Delta_{\mathrm{J}}$ & $\mathrm{kHz}$ & $0.2534(26)$ & $0.25439(34)$ & & \\
\hline$\Delta_{\mathrm{JK}}$ & $\mathrm{kHz}$ & $0.988(26)$ & $1.0094(12)$ & & \\
\hline$\Delta_{\mathrm{K}}$ & $\mathrm{kHz}$ & $0.54(15)$ & $0.532(23)$ & & \\
\hline$\delta_{\mathrm{J}}$ & $\mathrm{kHz}$ & $-0.01171(45)$ & $-0.011246(71)$ & & \\
\hline$\Delta_{\mathrm{K}}$ & $\mathrm{kHz}$ & $5.904(27)$ & $5.7985(37)$ & & \\
\hline$h_{K}$ & $\mathrm{kHz}$ & $-0.0040(34)$ & & & \\
\hline $\mathrm{D}_{\mathrm{pi} 2 J}$ & $\mathrm{MHz}$ & $0.1325(54)$ & & & \\
\hline $\mathrm{D}_{\mathrm{pi2K}}$ & $\mathrm{MHz}$ & $-1.03(19)$ & & & \\
\hline $\mathrm{D}_{\mathrm{pi2}-}$ & $\mathrm{MHz}$ & $-0.579(18)$ & & & \\
\hline$v_{3}$ & $\mathrm{~cm}^{-1}$ & $250.3(19)$ & & 235.3 & 15.0 \\
\hline $\mathrm{I}_{\alpha}$ & $\mathrm{u} \AA^{2}$ & $3.198(26)$ & $3.973(11)$ & & \\
\hline$\angle(\mathrm{i}, \mathrm{a})$ & $\circ$ & $34.113(96)$ & $31.753(79)$ & 35.40 & -1.71 \\
\hline$\angle(\mathrm{i}, \mathrm{b})$ & $\circ$ & $116.90(11)$ & $116.596(66)$ & 115.78 & 1.12 \\
\hline$\angle(\mathrm{i}, \mathrm{c})$ & $\circ$ & $70.641(99)$ & $73.943(35)$ & 67.50 & -3.14 \\
\hline $\mathrm{N}_{\mathrm{A}} / \mathrm{N}_{\mathrm{E}}^{\mathrm{c}}$ & & $59 / 57$ & $59 / 57$ & & \\
\hline$\sigma^{\mathrm{d}}$ & $\mathrm{kHz}$ & 17.6 & 1.4 & & \\
\hline
\end{tabular}

${ }^{a}$ Calculated at the MP2/6-311++G(d,p) level of theory

${ }^{b}$ Values obtained from the XIAM fit

${ }^{c}$ Number of the fitted A/E species transitions

${ }^{\mathrm{d}}$ Standard deviation of the fit 


\section{Table 2:}

Spectroscopic constants of methyl isobutyl ketone obtained with the program Erham in addition to Table 1 .

\begin{tabular}{lll}
\hline Parameter $^{\mathrm{a}}$ & Unit & Value \\
\hline$\rho$ & & $0.03331(12)$ \\
$\beta$ & $\circ$ & $10.719(33)$ \\
$\alpha$ & $\circ$ & $151.256(12)$ \\
$\epsilon_{10}$ & $\mathrm{GHz}$ & $-0.7941(10)$ \\
{$\left[\mathrm{G}_{\mathrm{a}}\right]_{10} \mathrm{~b}$} & $\mathrm{MHz}$ & $11.97(16)$ \\
{$\left[\mathrm{G}_{\mathrm{b}}\right]_{10} \mathrm{~b}$} & $\mathrm{MHz}$ & $-1.0080(37)$ \\
{$[\mathrm{A}-(\mathrm{B}+\mathrm{C}) / 2]_{10}$} & $\mathrm{MHz}$ & $0.1293(24)$ \\
{$[(\mathrm{B}+\mathrm{C}) / 2]_{10}$} & $\mathrm{kHz}$ & $-16.931(33)$ \\
\hline
\end{tabular}

${ }^{\text {a }}$ For the nomenclature of the parameters, see [16].

${ }^{b}$ The meaning of $\left[\mathrm{G}_{\mathrm{a}}\right]_{10}$ and $\left[\mathrm{G}_{\mathrm{b}}\right]_{10}$ is $(1,0,-1,0,0,1)$ and $(1,0,-1,1,0,0)$, respectively, in the (IQ1,IQ2,MEG,KAP,JP,KP) notation used in the Erham program instructions available in [17]. 


\section{Figure captions}

Fig. 1: A typical spectrum of methyl isobutyl ketone in the high resolution mode, typical line width from 9 to $22 \mathrm{kHz}$. For this spetrum, 59 FIDs were co-added.

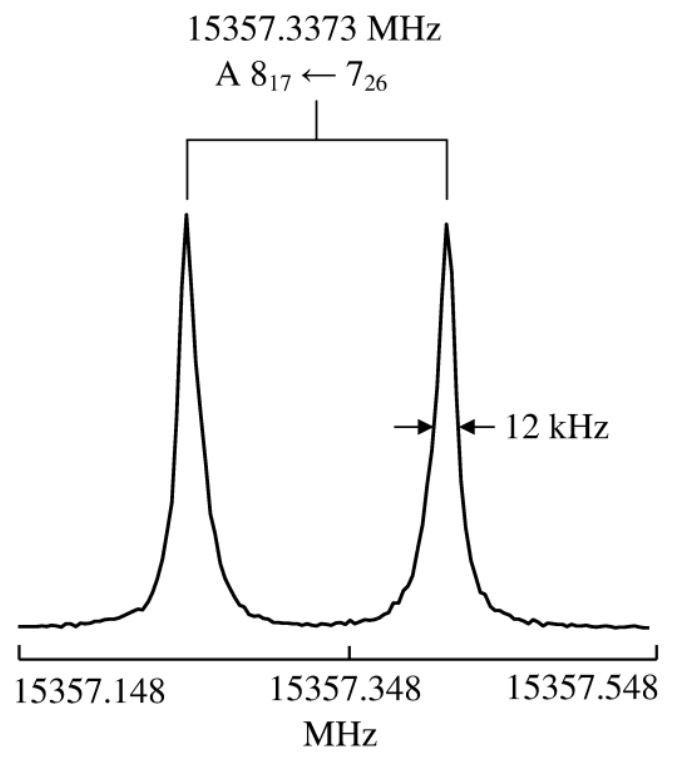

Fig. 2: The structures of conformer I of methyl isobutyl ketone calculated at the MP2/6$311++\mathrm{G}(\mathrm{d}, \mathrm{p})$ level of theory. The isopropyl group is tilted out of the $\mathrm{C}-(\mathrm{CO})-\mathrm{C}$ plane by an angle of $20.2^{\circ}$.
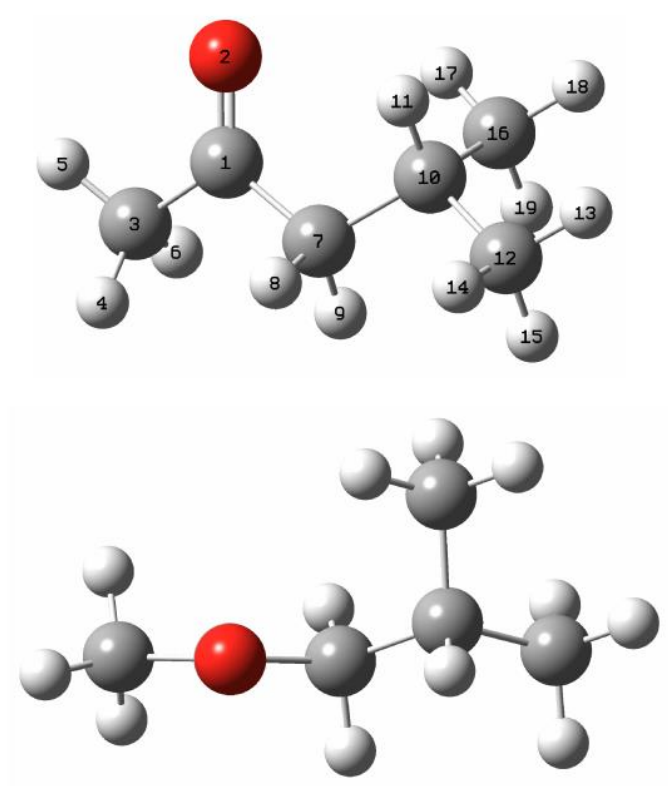

Conformer I (most stable) 
Fig. 3: Four conformers of methyl isobutyl ketone optimized with two different methods MP2 and B3LYP using the same basis set 6-311++G(d,p). The energies relative to the lowest energy conformer I $(E=-310.2466733$ Hartree and -311.1916584 Hartree for the MP2 and B3LYP methods, repectively) are given. Large energy differences between the calculations with the MP2 and B3LYP methods are found for conformer III and IV where the isopropyl group is completely out of the $\mathrm{C}-(\mathrm{CO})-\mathrm{C}$ plane.

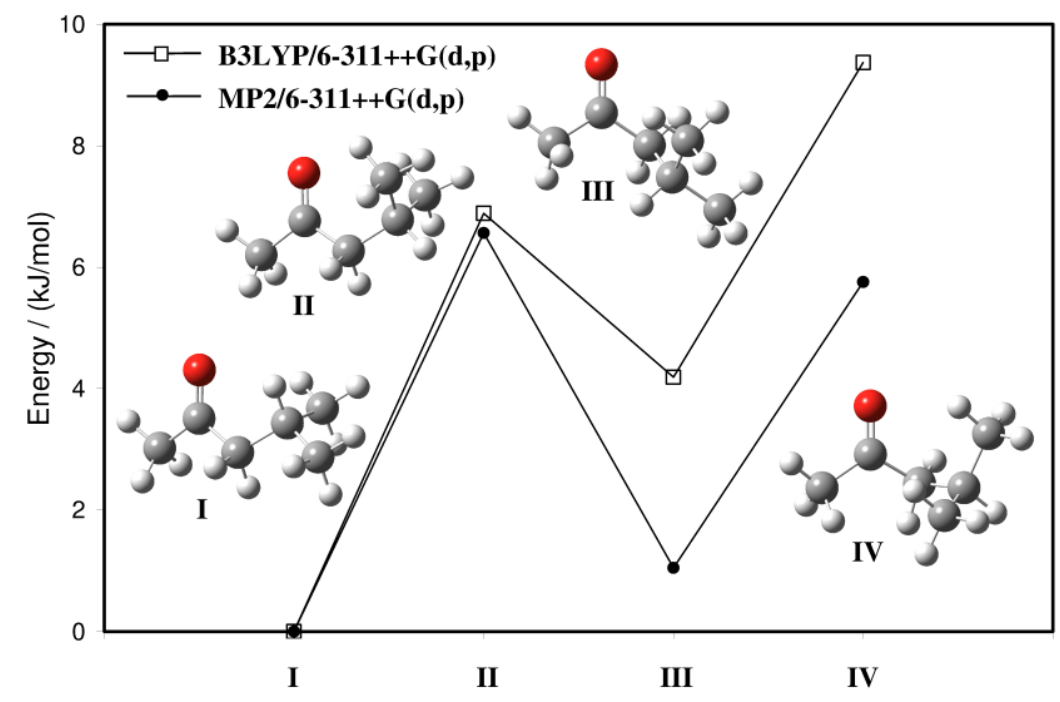

Fig. 4: The potential energy minimum path of methyl isobutyl ketone obtained by rotating the isopropyl group. The dihedral angle $\varphi_{1}=\angle\left(\mathrm{C}_{1}, \mathrm{C}_{7}, \mathrm{C}_{10}, \mathrm{C}_{12}\right)$ was fixed, while all other parameters were optimized. Two enantiomers of conformer I and conformer II were found in this path. For curve labeling see Figure 5.

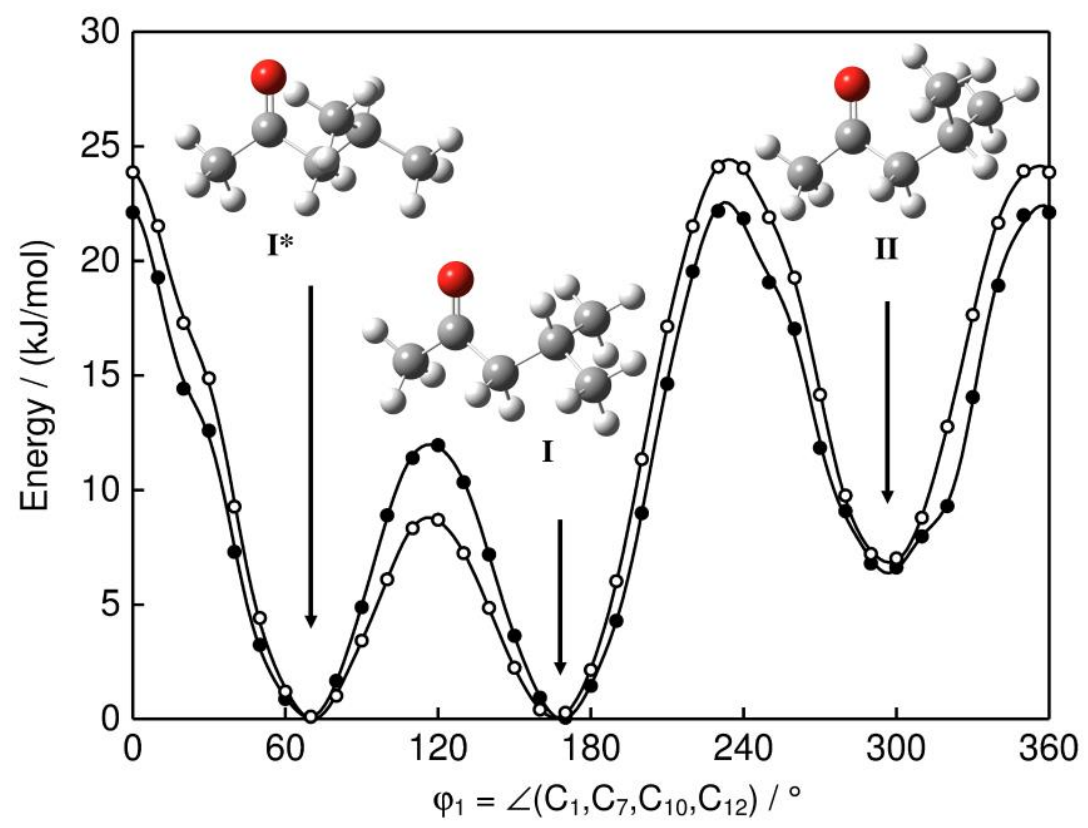


Fig. 5: The potential energy minimum path obtained by rotating the isobutyl group. In this case, the dihedral angle $\varphi_{2}=\angle\left(\mathrm{O}_{2}, \mathrm{C}_{1}, \mathrm{C}_{7}, \mathrm{C}_{10}\right)$ was fixed.

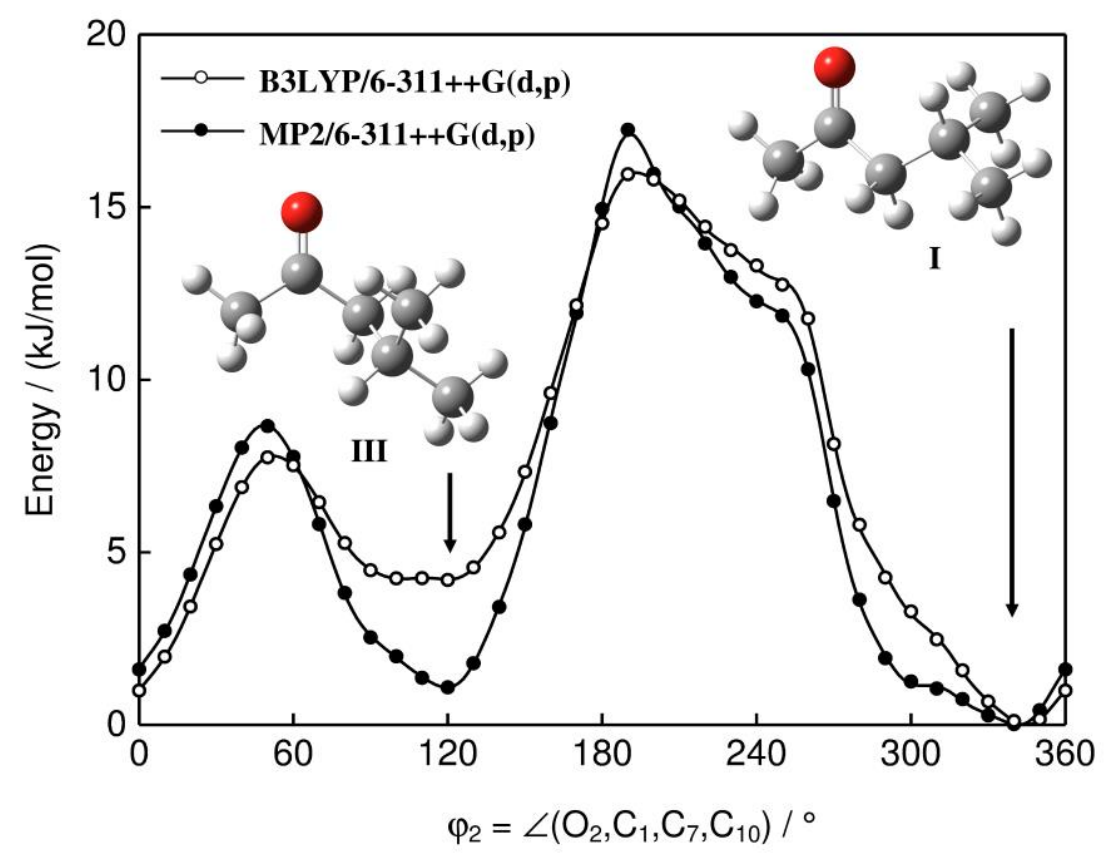

Fig. 6: A broadband scan of methyl isobutyl ketone from $15.1 \mathrm{GHz}$ to $17.0 \mathrm{GHz}$. The measured spectrum is shown on the upper trace. On the lower trace, the assigned A (in black) and $\mathrm{E}$ (in red) species lines are given and indicated without line intensities. An example of A and $\mathrm{E}$ species $c$-type Q-branches is also given on the lower trace.

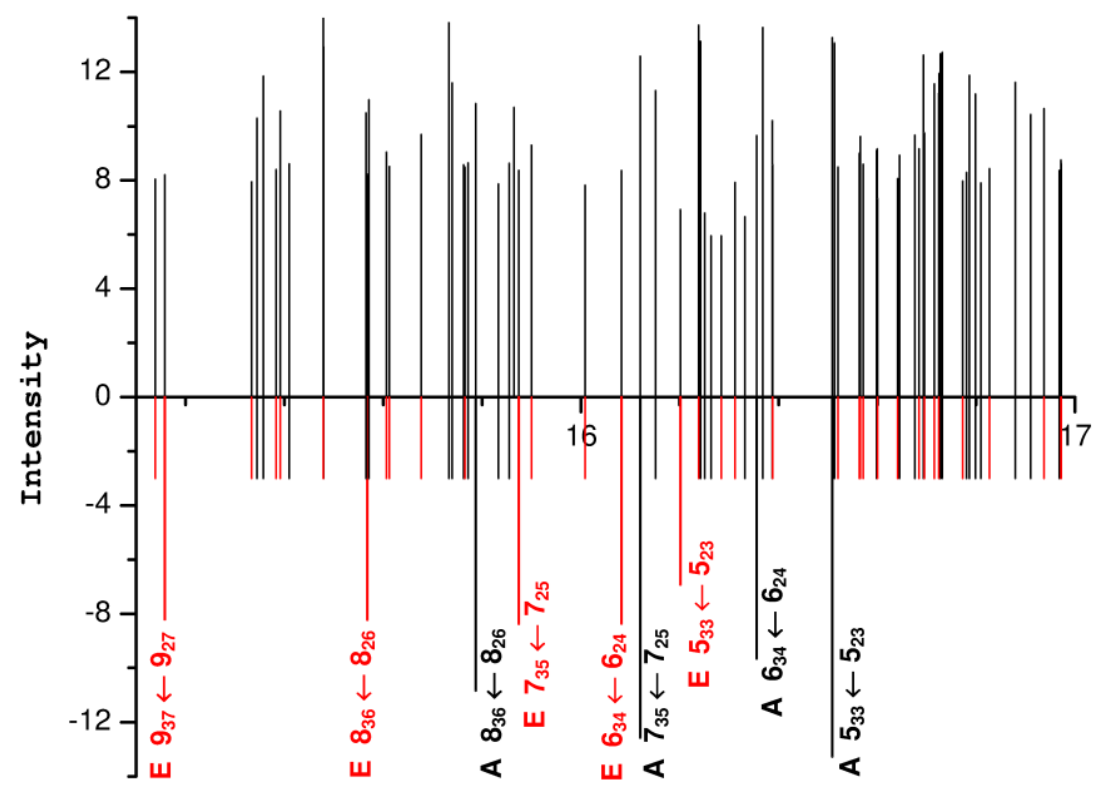

